OPEN ACCESS

Edited by:

Hongqiang Song,

Shandong University, China

Reviewed by:

Mingde Ding,

Nanjing University, China

Xiaoli Yan,

National Astronomical Observatories

(CAS), China

*Correspondence:

Haimin Wang

haimin.wang@njit.edu

Specialty section:

This article was submitted to

Stellar and Solar Physics,

a section of the journal

Frontiers in Astronomy and Space

Sciences

Received: 28 January 2019

Accepted: 11 March 2019

Published: 04 April 2019

Citation:

Wang H and Liu C (2019) Signatures of Magnetic Flux Ropes in the Low Solar Atmosphere Observed in High

Resolution

Front. Astron. Space Sci. 6:18. doi: 10.3389/fspas.2019.00018

\section{Signatures of Magnetic Flux Ropes in the Low Solar Atmosphere Observed in High Resolution}

\author{
Haimin Wang ${ }^{1,2 *}$ and Chang Liu ${ }^{1,2}$ \\ ${ }^{1}$ Big Bear Solar Observatory, New Jersey Institute of Technology, Big Bear City, CA, United States, ${ }^{2}$ Institute of Space \\ Weather Sciences, New Jersey Institute of Technology, Newark, NJ, United States
}

Magnetic flux ropes (MFRs) are important physical features closely related to solar eruptive activities with potential space weather consequences. Studying MFRs in the low solar atmosphere can shed light on their origin and subsequent magnetic structural evolution. In recent years, observations of solar photosphere and chromosphere reached a spatial resolution of 0.1 to 0.2 arcsec with the operation of meter class ground-based telescopes, such as the $1.6 \mathrm{~m}$ Goode Solar Telescope at Big Bear Solar Observatory and the $1 \mathrm{~m}$ New Vacuum Solar Telescope at Yunnan Observatory. The obtained chromospheric $\mathrm{H} \alpha$ filtergrams with the highest resolution thus far have revealed detailed properties of MFRs before and during eruptions, and the observed pre-eruption structures of MFRs are well consistent with those demonstrated by non-linear force-free field extrapolations. There is also evidence that MFRs may exist in the photosphere. The magnetic channel structure, with multiple polarity inversions and only discernible in high-resolution magnetograph observations, may be a signature of photospheric MFRs. These MFRs are likely formed below the surface due to motions in the convection zone and appear in the photosphere through flux emergence. Triggering of some solar eruptions is associated with an enhancing twist in the low-atmospheric MFRs.

Keywords: sun, flux rope, eruption, chromosphere, photosphere

\section{INTRODUCTION}

Magnetic flux ropes are generally defined as a bundle of magnetic fields that are twisted about each other and wrap around a common axis. These current-carrying magnetic field systems are crucially important as they may exhibit eruptive activities while being subject to different modes of instabilities and forces (Myers et al., 2017). Significant attention has been drawn to the structure and evolution of solar magnetic flux ropes, as they are believed to constitute the key component of coronal mass ejections (CMEs), a major form of solar eruptions that can have a direct impact on space weather (Filippov et al., 2015; Chen, 2017; Cheng et al., 2017). On the large scale, magnetic flux ropes are detected in the interplanetary magnetic clouds formed by CMEs (Burlaga et al., 1982), which may cause geomagnetic disturbances when interacting with Earth's magnetic field. In the solar corona, some studies suggest that flux ropes are produced via a magnetic reconnection between different branches of loops during the process of eruptions (e.g., Liu et al., 2010; Cheng et al., 2011; Green et al., 2011); however, there is also evidence that flux ropes pre-exist before eruptions as they may be built up gradually (e.g., Zhang et al., 2012; Cheng et al., 2014). Many models have also been developed to address the formation of magnetic 
flux ropes, such as via magnetic reconnection (e.g., van Ballegooijen and Martens, 1989; Amari et al., 2000; Priest and Longcope, 2017) and sunspot rotation (e.g., Yan et al., 2015). The genesis and evolution of solar magnetic flux ropes is therefore an intriguing and challenging question, the answer of which can benefit from a quantitative characterization of solar magnetic fields in terms of physical parameters (e.g., magnetic twist, electric current). This can now be routinely obtained by magnetic field modeling methods, such as the non-linear force-free field (NLFFF) extrapolations, based on the advanced vector magnetograph observations such as from Hinode and the Helioseismic and Magnetic Imager (HMI) on board the Solar Dynamics Observatory (SDO). Reconstructing coronal magnetic field enables not only the identification of magnetic flux ropes through computing magnetic twist of individual field lines and mapping magnetic connectivities, but also the scrutinization of flux rope evolution by monitoring their physical properties (Liu et al., 2016). A wealth of studies have since then explored the magnetic environment of flux ropes (e.g., Guo et al., 2010; Jiang et al., 2014) and attempted to understand successful vs. failed eruptions under the context of kink and torus instabilities (e.g., Liu et al., 2015, 2016; Amari et al., 2018; Jing et al., 2018).

Obviously, high-resolution observations can be invaluable in shedding light on the central question above, by visualizing flux rope structures and helping disentangle their relation to eruptions. However, previous research has primarily relied on spaceborne coronal observations with moderate resolution. It is notable that enormous advances in revealing and studying the fine-scale structures and dynamics of the low solar atmosphere has been achieved thanks to the recent development of large ground-based solar telescopes that produce observations with highest spatiotemporal resolution available thus far. These
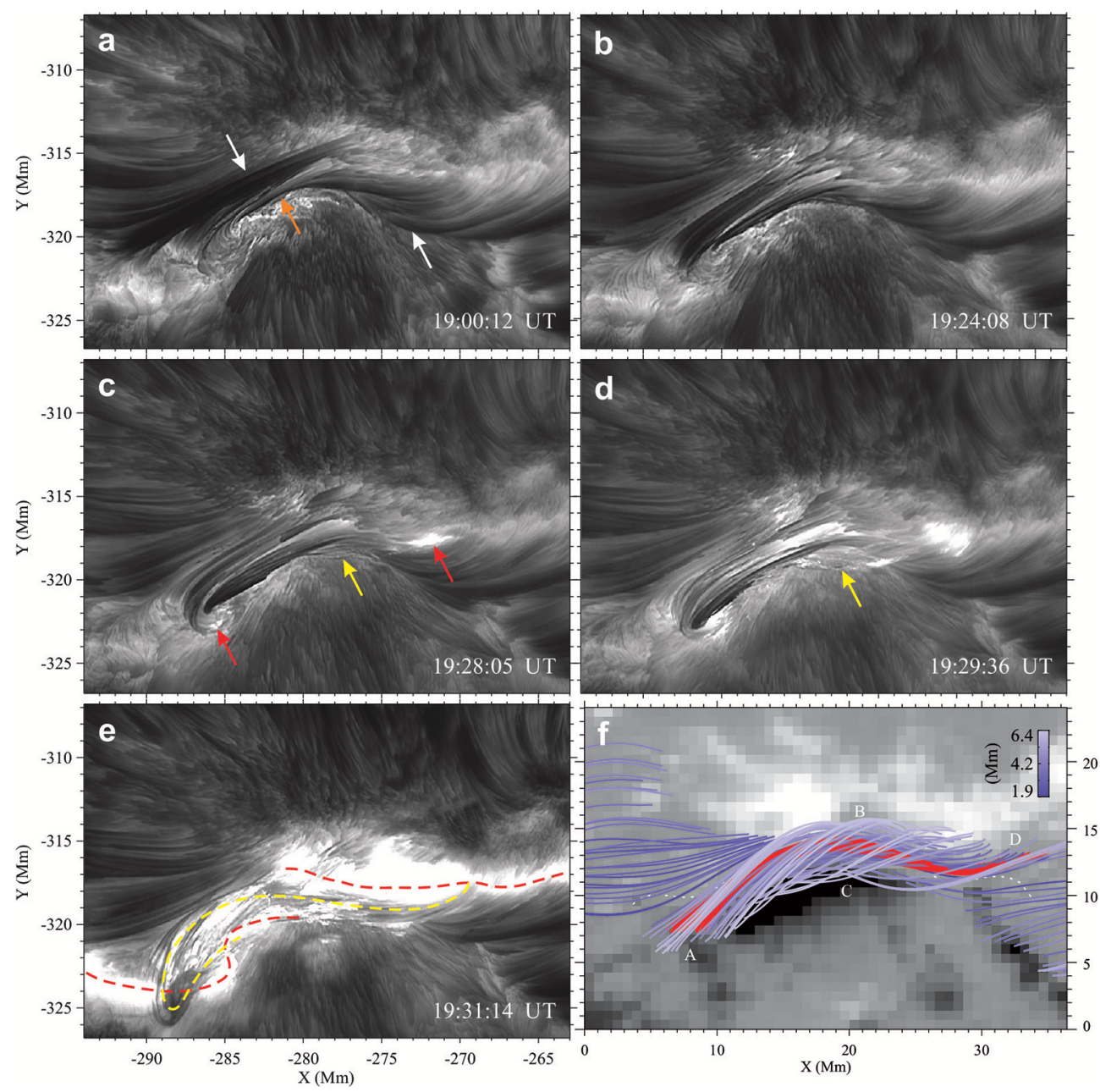

FIGURE 1 | BBSO/GST H $\alpha$ time-sequence observations (a-e) and NLFFF modeling (f) of a magnetic flux rope in NOAA active region 11817 on 2013 August 11. The white (orange) arrows point to the weakened (enhanced) loops at the event onset, and the red arrows indicate the initial footpoint brightening. The yellow arrows and dashed line delineate the active flux rope in motion, and the red dashed line mark the induced double ribbons of a C2.1 flare. The plotted representative field lines are from a preflare optimization NLFFF extrapolation model, which is based on the SDO/HMl vector magnetic field data remapped with a Lambert cylindrical equal area projection. The twisted flux rope in red (with a maximum height $\lesssim 4.0 \mathrm{Mm}$ ) is embedded in sheared arcade fields (largely AB and CD) in blue (with a maximum height $\lesssim 6.4 \mathrm{Mm}$ ) (Wang et al., 2015). Credit: Nature Communications. 
include the Swedish $1 \mathrm{~m}$ Solar Telescope on La Palma, $1.6 \mathrm{~m}$ Goode Solar Telescope (GST; Goode et al., 2010) at Big Bear Solar Observatory (BBSO), $1.5 \mathrm{~m}$ solar telescope GREGOR on Tenerife, and $1 \mathrm{~m}$ New Vacuum Solar Telescope (NVST; Liu et al., 2014) at Yunnan Observatory. In this review, we highlight the most important observational signatures of magnetic flux ropes in the chromosphere as seen by BBSO/GST and NVST, and, remarkably, even in the photosphere as seen by BBSO/GST. We also discuss the future prospects and challenges in this important research field.

\section{CHROMOSPHERIC SIGNATURES OF FLUX ROPES}

The key advancement in the observation of magnetic flux ropes has been made using $\mathrm{H} \alpha$ filtergrams with a spatial resolution in the order of $0.1^{\prime \prime}$ and a temporal cadence of 10-20 s. The twisted magnetic structure of flux ropes in $\mathrm{H} \alpha$ is discernible before eruptive activities and becomes much more obvious during the process of their activation or eruption. Using BBSO/GST $\mathrm{H} \alpha$ observations, Wang et al. (2015) clearly demonstrated that a bunch of flux loops seemingly peeled off from an inverse S-shaped flux system to unveil itself as a twisted flux rope and meanwhile induced a two-ribbon C2.1 flare (Figures 1a-e). This marks the first witness of the structural evolution of a flux rope in the chromosphere. NLFFF extrapolation models (constructed with the optimization method) suggest that sheared arcades embed this twisted flux rope (Figure 1f), which evolves to an unstable state as its twist enhances (and thus subjects to the helical kink instability) but ultimately fails to erupt due to the strong confinement of the ambient strapping field. Liu et al. (2016) further showed that such a pre-eruption enhancement of a magnetic twist also occurred for other homologous events, either confined or eruptive, in this active region.

It is generally considered that filaments are a reliable proxy of flux ropes. Since its operation, NVST has produced excellent observations of filament activities. As a good example, Li et al. (2017) studied sympathetic, failed eruptions of two filaments near the east limb with NVST $\mathrm{H} \alpha$ observations (Figures 2a,b) and NLFFF extrapolations (constructed with the flux rope insertion method; Figure 2c). The authors attributed the activation of the left filament to kink instability, and that of the right filament to the weakening of overlying fields following an induced Xpoint reconnection between the filaments. Notably, they found a clear rotational motion of the southern portion of both filaments, which is likely to indicate an untwisting of the erupting filaments. In another study, Yang et al. (2014) observed that a filament is activated by magnetic field cancellation and undergoes a failed eruption, during the process of which it clearly tracks a twisted flux rope structure.

The above studies mainly deal with the evolution of flux ropes that already exist. Importantly, high-resolution observations are powerful in disclosing the complete dynamic evolution of magnetic flux ropes related to the initiation of flares/CMEs. Using BBSO/GST H $\alpha$ data, Yan et al. (2017) presented that a smallscale flux rope (Figures $\mathbf{2 d - h}$ ), with its footpoints showing a rotational motion, emerges near a large sunspot and subsequently erupts, driving an M1.0 flare and a CME. The presence of the flux rope was evidenced by both optimization NLFFF (Figure 2i) and data-driven magnetohydrodynamic modeling. Kumar et al. (2015) reported that a small, twisted chromospheric flux rope is formed between two sheared J-shaped $\mathrm{H} \alpha$ loops by magnetic reconnection, which is associated with flux cancellation and shear flows and causes an M1.0 flare (Figure 3, upper six panels). Jet activities and cool plasma inflow are also observed at the reconnection site. Kumar et al. (2017) further showed another clear example of the formation of a small unstable, rotating flux rope from a series of magnetic reconnection between chromospheric $\mathrm{H} \alpha$ loops together with very rapid flux cancellation. Such a formation process of flux ropes via the tether-cutting-like reconnection accompanied by magnetic cancellations was also observed by Xue et al. (2017) using NVST H $\alpha$ data. The authors detailed various signatures of the reconnection between two branches of highly sheared arcades, and found that the central part of the newly formed long flux rope connecting two far footpoints bears a concave-up-shape structure with many fine threads; the expected another sets of shorter loops are also seen (Figure 3, lower six panels). For the sake of completeness, we note that NVST $\mathrm{H} \alpha$ images display twisted structures of two active-region eruptive filaments in Yan et al. (2015), where the authors identified the filaments as magnetic flux ropes with NLFFF modeling and suggested that they are formed after horizontal magnetic fields are twisted as a result of sunspot rotation.

\section{PHOTOSPHERIC SIGNATURES OF FLUX ROPES}

The above chromospheric observations evince that flux ropes can be formed due to magnetic reconnection above the surface, and also that they might emerge from below the surface, in which case flux ropes must be formed in the solar convection zone. In retrospect, one of the pioneering studies of the intrinsic relationship between occurrence of flares and evolution of photospheric magnetic configuration was conducted by Tanaka (1991) to infer the subsurface magnetic field structure of a flare-productive $\delta$-spot group. The characteristic pattern of the unusually fast evolution of this sunspot group consists of a shearing phase when spots grow and a shear reduction phase when spots decay. This observation led the author to construct a cartoon model of a complex magnetic system comprising twisted magnetic knots and a long-winding flux rope, the consecutive emergence of which was shown to be consistent with the abnormal evolution of the flaring active region of interest. This approach was adopted and further explored by studies such as Ishii et al. (1998) and Kurokawa et al. (2002), in which the authors schematically illustrated that the emergence of twisted magnetic flux ropes may explain the observed proper motions of sunspots and other drastic evolution of magnetic structures, and may be the source of strong flaring activities.

Along the same line, Zirin and Wang (1993) made the discovery that channel-like magnetic structures were formed 


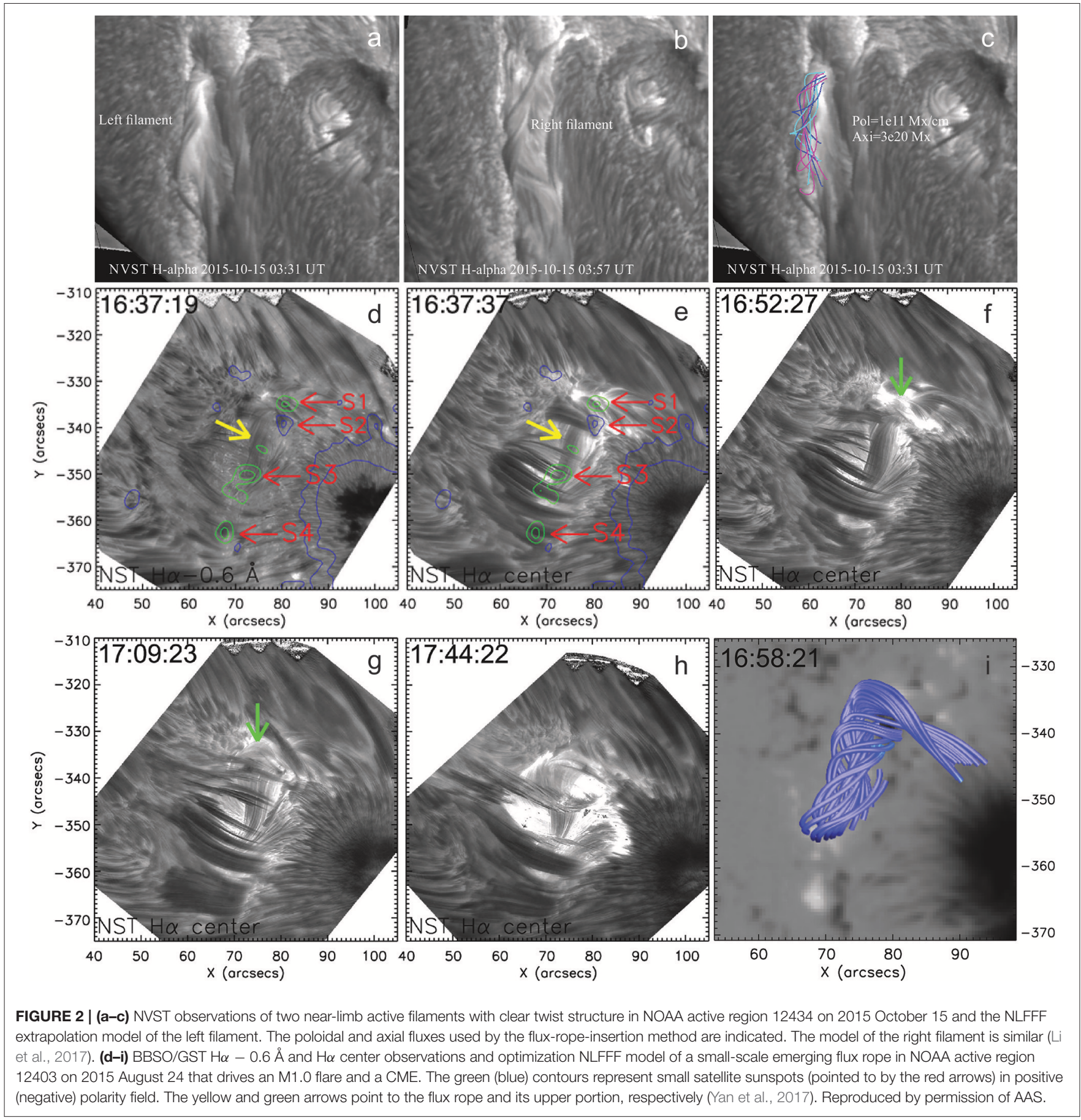

when magnetic fluxes emerged inside the common penumbra of a $\delta$-spot group. These magnetic channels on the surface take the form of elongated, opposite-polarity flux system with multiple polarity inversions, along which there are strong transverse magnetic field and surface flows. Using observations from Hinode (which provides magnetic field measurement with the highest resolution before the $\mathrm{BBSO} / \mathrm{GST}$ era) and with the aid of NLFFF extrapolation, several studies analyzed magnetic channels in detail and suggested that they may originate from the emergence of twisted flux tubes from below the surface (e.g., Kubo et al., 2007; Wang et al., 2008; Lim et al., 2010). This view is supported by recent theoretical modeling, which has been able to reveal detailed flux emergence process in relation to the generation of magnetic channels. Toriumi and Takasao (2017) carried out a series of flux emergence modeling, among which an emerging highly twisted, kink-unstable flux tube from the convection zone to the corona is employed to simulate the formation of a compact $\delta$-spot group. It was shown 


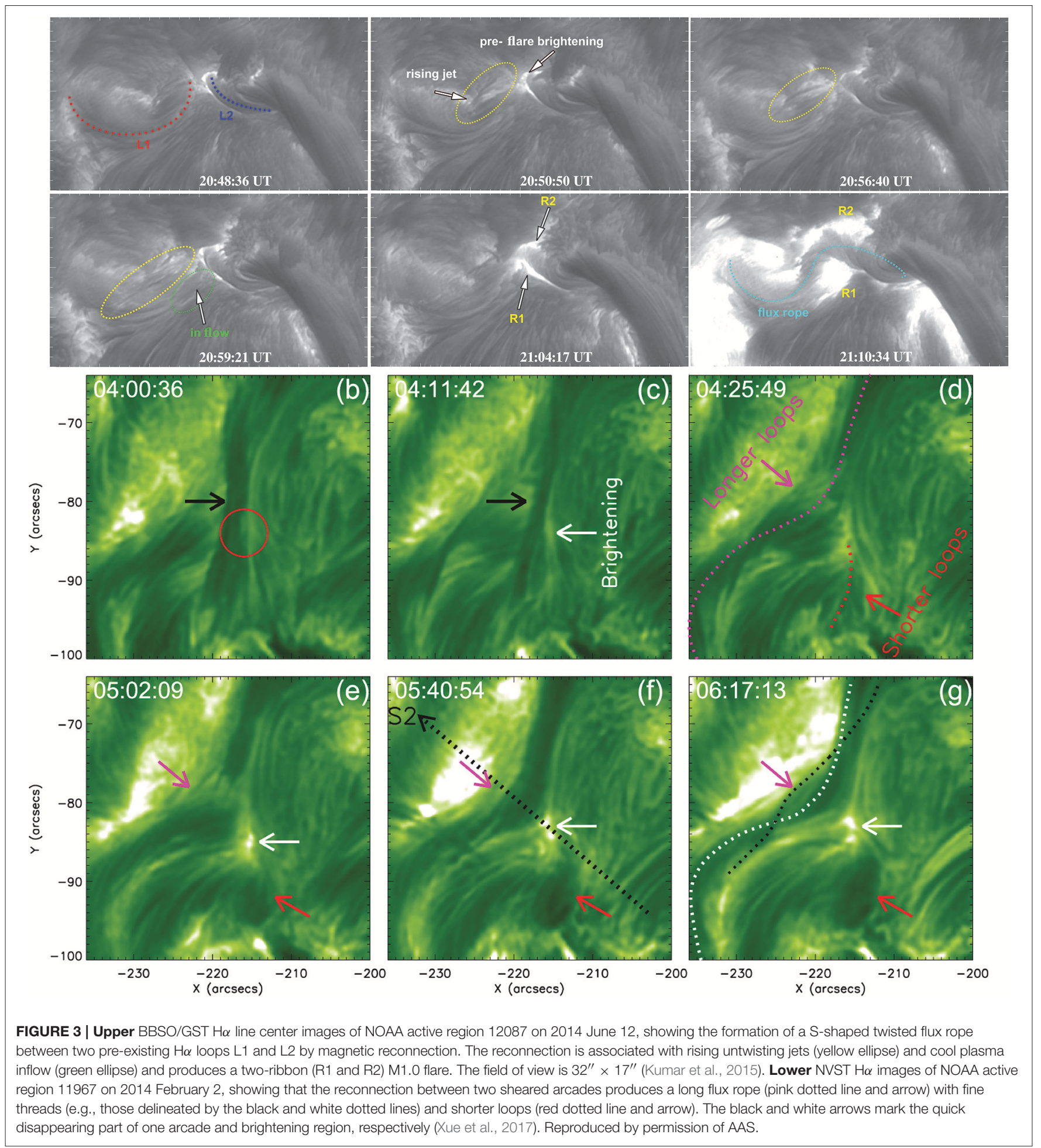

that as magnetic fields are advected, stretched, and compressed during their emergence, a highly sheared magnetic polarity inversion line (PIL) of the $\delta$ spot is formed with an elongated, alternating pattern of both polarities, which is greatly reminiscent of magnetic channels. The simulation results of Knizhnik et al.
(2018) corroborate the kink-unstable emerging flux ropes as a promising mechanism of $\delta$ spot formation, and their results also showed the development of elongated, secondary oppositepolarity regions between primary polarities, which are similar to the observed magnetic channels. Therefore, it is appealing to 
suggest that magnetic channels are a photospheric signature of emerging flux ropes.

Previous studies have shown that high spatial resolution and high polarimetry accuracy are required to observe the magnetic channel structure in detail. Meeting these properties, BBSO/GST can provide vector magnetograms at $0.24^{\prime \prime}$ resolution and up to about $30 \mathrm{~s}$ cadence through the spectropolarimetric observations of the Fe I $1564.8 \mathrm{~nm}$ line with the Near InfraRed Imaging Spectropolarimeter (NIRIS). The unprecedented high spatiotemporal resolution NIRIS vector field data allowed Wang et al. (2017) to not only detect a magnetic channel not discernible with SDO/HMI but also study its temporal evolution with related to the flare occurrence in an extended active region with the $\delta$ configuration (Figures $4 \mathbf{4 a}, \mathbf{b}$ ). The authors found that the channel structure at the PIL is located near the footpoints of sheared arcades, and its strengthening in terms of enhancement of magnetic fluxes and currents is cotemporal with episodes of flare precursor brightening observed close by (Figure 4c).

As emerging magnetic fluxes on the surface carry signatures of the pre-existing flux ropes, some photospheric appearance of flux ropes may also be reflected in white light observations, especially at high resolution. BBSO/GST's Broad-Band Filter Imager provides direct imaging in the broadband $\mathrm{TiO}$ (a proxy for continuum in photosphere at $705.7 \mathrm{~nm}$ ) at $0.1^{\prime \prime}$ resolution and $15 \mathrm{~s}$ cadence, which has been shown to be another effective diagnosis of photospheric flux ropes. Sharykin et al. (2017) reported $\mathrm{TiO}$ flux rope structures observed along
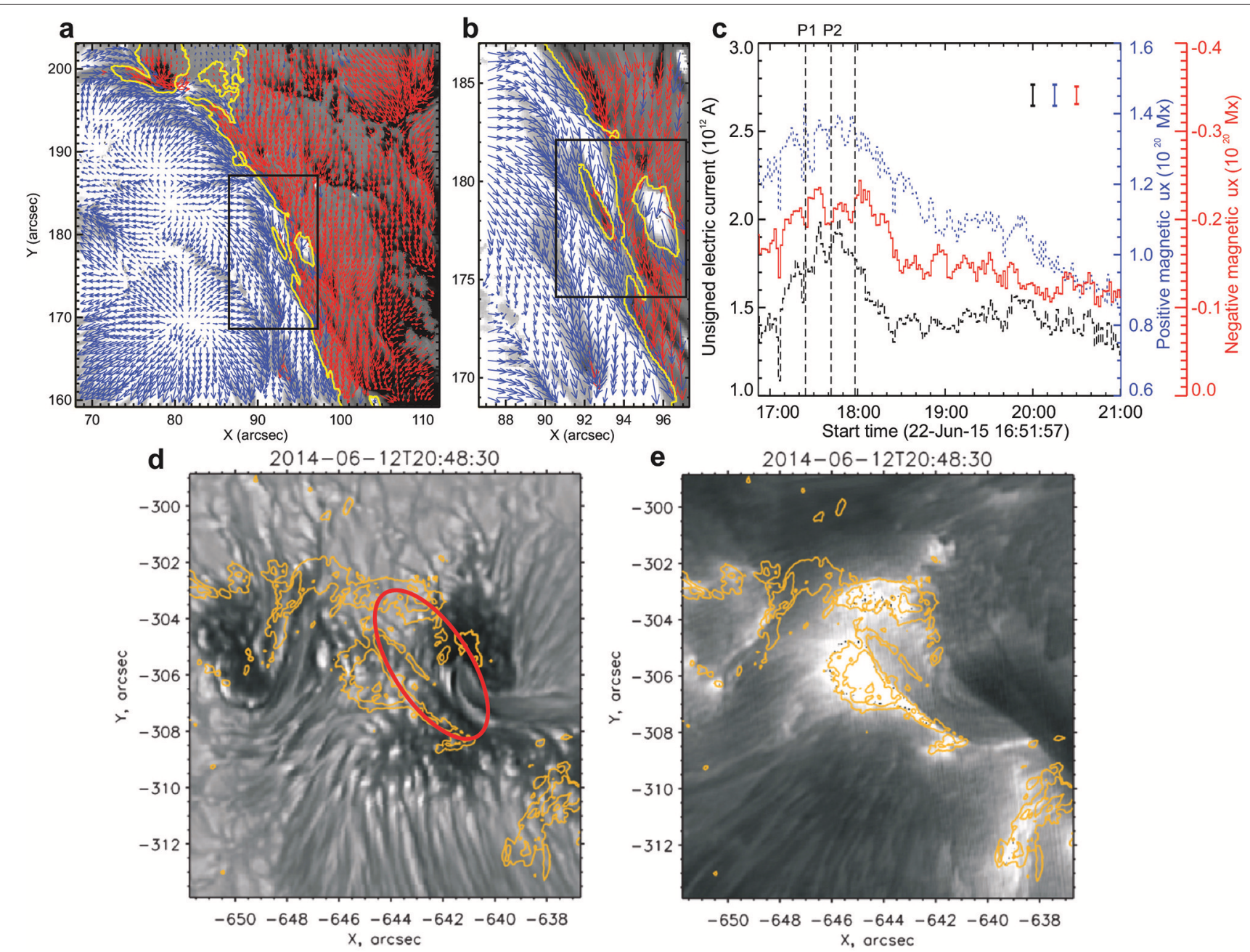

FIGURE 4 | Upper three panels: BBSO/GST near-infrared vector magnetic field map of NOAA AR 12371 on 2015 June 22 (a), the identified magnetic channel structure at the PIL [b; a subregion denoted by the box in (a)], and time profiles of total positive (blue) and negative (red) magnetic fluxes and the unsigned electric current (black), calculated over the boxed region in (b). The three vertical dashed lines in (c) mark the times of precursor episodes P1 and P2 and the peak time of the flare nonthermal emission, respectively (Wang et al., 2017). Credits: Nature Astronomy. Lower two panels: Photospheric manifestations of flux ropes seen as a sheared arcade along the PIL (marked by the red ellipse in d), as observed in broadband TiO with BBSO/GST in NOAA active region 12087 on 2014 June 12 . Besides the double ribbons of the confined $\mathrm{M} 1.0$ flare in $\mathrm{H} \alpha$ line core (e), a third thread-like ribbon in $\mathrm{H} \alpha$ red wing (yellow contours) is cospatial with the flux rope structure (Sharykin et al., 2017). Reproduced by permission of AAS. 
the PIL as a compact sheared arcade, which corresponds to twisted structures in $\mathrm{H} \alpha$ line core and a third tiny thread-like ribbon in $\mathrm{H} \alpha$ red wing besides the double ribbons of a confined M1.0 flare (Figures 4d,e). After combining analyses of photospheric flows and magnetic field topology, the authors suggested that interacting flux ropes form an elongated current sheet along the PIL to trigger the flare. Thus far, the most prominent signatures of possible flux ropes observed at the photospheric level was described by Wang et al. (2018b) for the famous NOAA active region 12673 in September 2017. Within the light bridge sections of the $\delta$-spot groups, the authors observed alternating bright-dark spiral structures in $\mathrm{TiO}$ and showed evidence that these structures possess strong magnetic fields with a surprising magnitude above $\sim 5,500 \mathrm{G}$ (Figure 5). The unusual magnetic property of this possibly twisted flux rope structure right at the photosphere may be responsible for the strong activities in this region including the X9.3 flare on 2017 September 6 and the X8.2 limb flare on September 10. For future investigations, we caution that photospheric images should be combined with other diagnoses to give a definite identification of photospheric flux rope structures. Magnetic field extrapolations certainly can be helpful, while the height of extrapolated fields need to be considered.

\section{DISCUSSION AND FUTURE PROSPECTIVE}

Flux ropes are one of the most important targets in solar physics research due to their potential space weather effects caused by their eruptions. With the operation of meter class groundbased telescopes, significant advancements have been recently made in detecting signatures of flux ropes in the chromosphere and photosphere and studying their structures during eruptive activities. It is anticipated that more detailed and clearer dynamic structures of flux ropes will be revealed when the $4 \mathrm{~m}$ Daniel K. Inouye Solar Telescope (DKIST) is online (Tritschler et al., 2016). Additional spectroscopy tools will also provide accurate measurement of magnetic and velocity fields, which are critical in quantitatively understanding the physical mechanism that drives the flux rope evolution.

As demonstrated in previous studies, the topological structure observed in $\mathrm{H} \alpha$ may provide hints on the magnetic twist of flux ropes. With higher resolution observations and advanced machine learning techniques, it is expected that such a twist can be quantitatively examined by tracing $\mathrm{H} \alpha$ features (Aschwanden et al., 2016). The obtained results can also be compared with those deduced from magnetic field extrapolation models. Characterizing magnetic twist within and properties of strapping

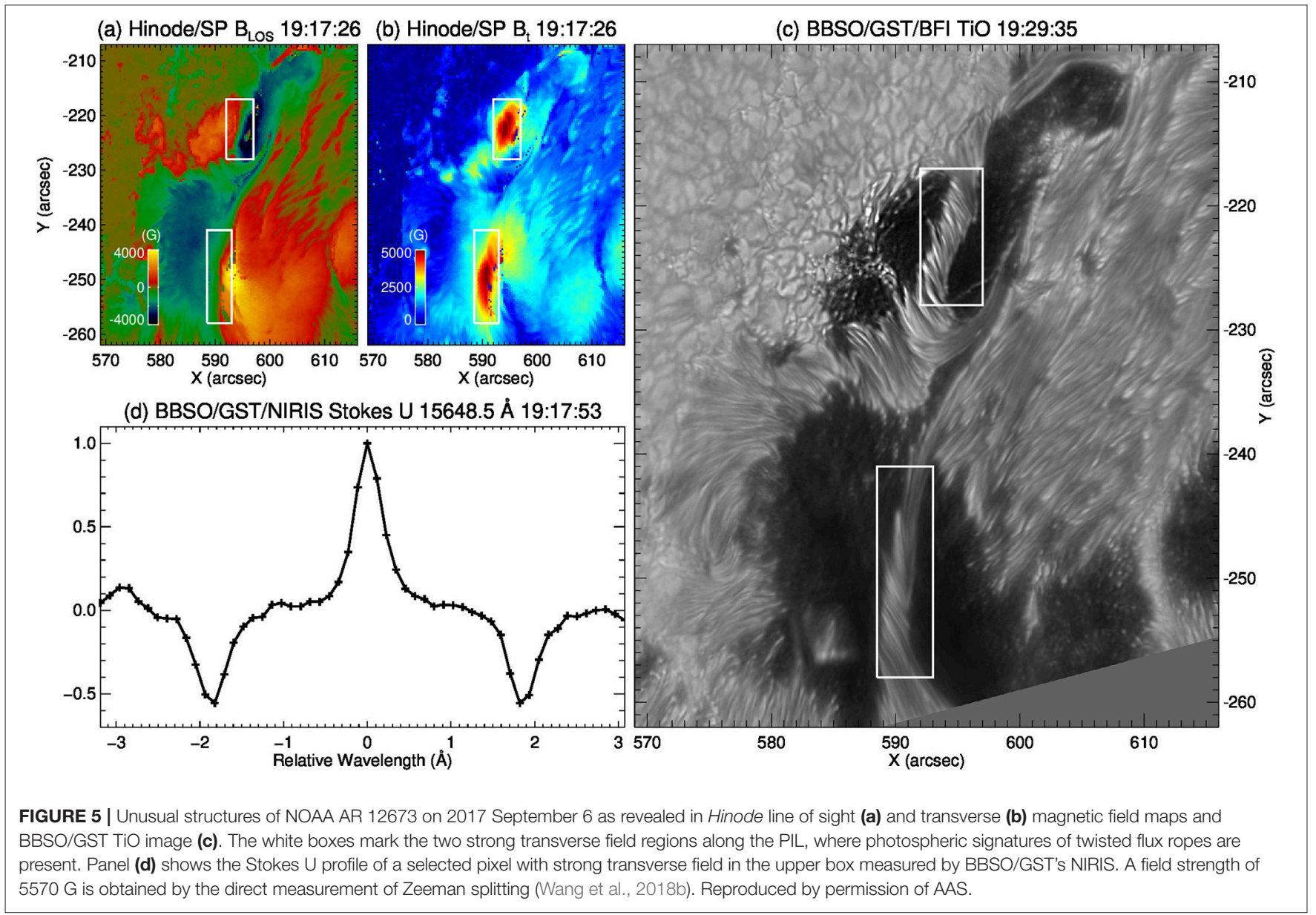


field above flux ropes will be important for understanding the kink and torus instabilities related to the onset of flux rope eruptions (Jing et al., 2018).

Beyond flux rope tracing, a direct measurement of vector magnetic field in flux ropes and filaments is highly desired. Spectropolarimetric observations using the He I $1083.0 \mathrm{~nm}$ line can provide a very useful diagnosis of magnetic field and flows in filaments and prominences. Hanaoka and Sakurai (2017) used data from full-disk 1083.0 spectropolarimetry to obtain a statistic evaluation of magnetic field orientation in filaments. Sasso et al. (2014) analyzed the 1083.0 spectropolarimetric observation of an active region filament activated by a flare and found evidence of a coronal flux rope. High-resolution observations in $1083.0 \mathrm{~nm}$ and corresponding advanced data analysis tools are being developed for DKIST. In addition to the $\mathrm{He}$ I $1083.0 \mathrm{~nm}$ line, spectral diagnostics using the chromospheric lines (e.g., the $\mathrm{Ca}$ II line at $854 \mathrm{~nm}$ and $\mathrm{H} \alpha$ line from BBSO/GST's Fast-Imaging Solar Spectrograph) in combination with other lines formed from the chromosphere to corona (observed with the Interface Region Imaging Spectrograph) can provide valuable information about flux rope formation especially the reconnection process (e.g., Cheng et al., 2015). Furthermore, studying the linkage of filaments to the photosphere by investigating their barb structure and anchoring footpoints observed in high resolution is also important for understanding the filament stability and related dynamics (e.g., Kuckein et al., 2016; Wang et al., 2018a).

\section{REFERENCES}

Amari, T., Canou, A., Aly, J.-J., Delyon, F., and Alauzet, F. (2018). Magnetic cage and rope as the key for solar eruptions. Nature 554, 211-215. doi: $10.1038 /$ nature24671

Amari, T., Luciani, J. F., Mikic, Z., and Linker, J. (2000). A twisted flux rope model for coronal mass ejections and two-ribbon flares. Astrophys. J. Lett. 529, L49-52. doi: 10.1086/312444

Aschwanden, M. J., Reardon, K., and Jess, D. B. (2016). Tracing the chromospheric and coronal magnetic field with AIA, IRIS, IBIS, and ROSA data. Astrophys. J. 826:61. doi: 10.3847/0004-637X/826/1/61

Burlaga, L. F., Klein, L., Sheeley N. R. Jr., Michels, D. J., Howard, R. A., Koomen, M. J., et al. (1982). A magnetic cloud and a coronal mass ejection. Geophys. Res. Lett. 9, 1317-1320. doi: 10.1029/GL009i012p01317

Chen, J. (2017). Physics of erupting solar flux ropes: coronal mass ejections (CMEs)-Recent advances in theory and observation. Phys. Plasmas 24:090501. doi: 10.1063/1.4993929

Cheng, X., Ding, M. D., and Fang, C. (2015). Imaging and spectroscopic diagnostics on the formation of two magnetic flux ropes revealed by SDO/AIA and IRIS. Astrophy. J. 804:82. doi: 10.1088/0004-637X/804/2/82

Cheng, X., Ding, M. D., Guo, Y., Zhang, J., Vourlidas, A., Liu, Y. D., et al. (2014). Tracking the evolution of a coherent magnetic flux rope continuously from the inner to the outer corona. Astrophy. J. 780:28. doi: 10.1088/0004-637X/780/1/28

Cheng, X., Guo, Y., and Ding, M. (2017). Origin and structures of solar eruptions I: magnetic flux rope. Sci. China Earth Sci. 60, 1383-1407. doi: 10.1007/s11430-017-9074-6

Cheng, X., Zhang, J., Liu, Y., and Ding, M. D. (2011). Observing flux rope formation during the impulsive phase of a solar eruption. Astrophys. J. Lett. 732:L25. doi: 10.1088/2041-8205/732/2/L25

Cheung, M. C. M., Rempel, M., Title, A. M., and Schüssler, M. (2010). Simulation of the formation of a solar active
Finally, the data-based magnetohydrodynamic modeling in high resolution will certainly advance our knowledge of flux ropes and their evolution from the lower to the upper solar atmosphere (Chen, 2017). For example, it may help eventually elucidate different scenarios, whether a flux rope is formed below the surface and brought up during flux emergence or is formed above the photosphere via such as magnetic reconnection. Recent high-resolution modeling has begun to make significant progress toward a more realistic framework for simulating the formation of complex active regions including their many fine-scale structures (e.g., light bridges, magnetic channels) during the emerging process of twisted magnetic field (e.g., Cheung et al., 2010; Toriumi and Takasao, 2017). We believe that joint efforts of highresolution modeling and observations will yield breakthroughs in understanding the structure, evolution, and eruption of magnetic flux ropes.

\section{AUTHOR CONTRIBUTIONS}

HW initiated the writing of the paper. CL revised the paper. Both studied related literatures.

\section{FUNDING}

We acknowledge the funding support by NASA grants NNX16AF72G, 80NSSC17K0016, 80NSSC18K0673, and 80NSSC18K1705, and by NSF grants AGS 1408703 and 1821294.

region. Astrophys. J. 720, 233-244. doi: 10.1088/0004-637X/ $720 / 1 / 233$

Filippov, B., Martsenyuk, O., Srivastava, A. K., and Uddin, W. (2015). Solar magnetic flux ropes. J. Astrophys. Astron. 36, 157-184. doi: 10.1007/s12036-015-9321-5

Goode, P. R., Yurchyshyn, V., Cao, W., Abramenko, V., Andic, A., Ahn, K., and Chae, J. (2010). Highest resolution observations of the quietest sun. Astrophys. J. Lett. 714, L31-L35. doi: 10.1088/2041-8205/714/1/L31

Green, L. M., Kliem, B., and Wallace, A. J. (2011). Photospheric flux cancellation and associated flux rope formation and eruption. Astron. Astrophys. 526:A2. doi: 10.1051/0004-6361/201015146

Guo, Y., Schmieder, B., Démoulin, P., Wiegelmann, T., Aulanier, G., Török, T., et al. (2010). Coexisting flux rope and dipped arcade sections along one solar filament. Astrophys. J. 714, 343-354. doi: 10.1088/0004-637X/ $714 / 1 / 343$

Hanaoka, Y., and Sakurai, T. (2017). Statistical study of the magnetic field orientation in solar filaments. Astrophys. J. 851:130. doi: 10.3847/1538-4357/aa9cf1

Ishii, T. T., Kurokawa, H., and Takeuchi, T. T. (1998). Emergence of a twisted magnetic flux bundle as a source of strong flare activity. Astrophys. J. 499, 898-904.

Jiang, C., Wu, S. T., Feng, X., and Hu, Q. (2014). Nonlinear force-free field extrapolation of a coronal magnetic flux rope supporting a large-scale solar filament from a photospheric vector magnetogram. Astrophys. J. Lett. 786:L16. doi: 10.1088/2041-8205/786/2/L16

Jing, J., Liu, C., Lee, J., Ji, H., Liu, N., Xu, Y., et al. (2018). Statistical analysis of torus and kink instabilities in solar eruptions. Astrophys. J. 864:138. doi: 10.3847/1538-4357/aad6e4

Knizhnik, K. J., Linton, M. G., and DeVore, C. R. (2018). The role of twist in kinked flux rope emergence and delta-spot formation. Astrophys. J. 864:89. doi: $10.3847 / 1538-4357 /$ aad68c 
Kubo, M., Yokoyama, T., Katsukawa, Y., Lites, B., Tsuneta, S., Suematsu, Y., et al. (2007). Hinode observations of a vector magnetic field change associated with a flare on 2006 December 13. Public. Astron. Soc. Jpn. 59, S779-S784. doi: 10.1093/pasj/59.sp3.S779

Kuckein, C., Verma, M., and Denker, C. (2016). Giant quiescent solar filament observed with high-resolution spectroscopy. Astron. Astrophys. 589:A84. doi: 10.1051/0004-6361/201526636

Kumar, P., Yurchyshyn, V., Cho, K.-S., and Wang, H. (2017). Multiwavelength observations of a flux rope formation by series of magnetic reconnection in the chromosphere. Astron. Astrophys. 603:A36. doi: 10.1051/0004-6361/201629295

Kumar, P., Yurchyshyn, V., Wang, H., and Cho, K.-S. (2015). Formation and eruption of a small flux rope in the chromosphere observed by NST, IRIS, and SDO. Astrophys. J. 809:83. doi: 10.1088/0004-637X/809/1/83

Kurokawa, H., Wang, T., and Ishii, T. T. (2002). Emergence and drastic breakdown of a twisted flux rope to trigger strong solar flares in NOAA active region 9026. Astrophys. J. 572, 598-608. doi: 10.1086/340305

Li, S., Su, Y., Zhou, T., van Ballegooijen, A., Sun, X., and Ji, H. (2017). Highresolution observations of sympathetic filament eruptions by NVST. Astrophys. J. 844:70. doi: 10.3847/1538-4357/aa78f5

Lim, E.-K., Chae, J., Jing, J., Wang, H., and Wiegelmann, T. (2010). The formation of a magnetic channel by the emergence of current-carrying magnetic fields. Astrophys. J. 719, 403-414. doi: 10.1088/0004-637X/719/1/403

Liu, C., Deng, N., Liu, R., Lee, J., Pariat, É., Wiegelmann, T., et al. (2015). A circularribbon solar flare following an asymmetric filament eruption. Astrophys. J. Lett. 812:L19. doi: 10.1088/2041-8205/812/2/L19

Liu, R., Kliem, B., Titov, V. S., Chen, J., Wang, Y., Wang, H., et al. (2016). Structure, stability, and evolution of magnetic flux ropes from the perspective of magnetic twist. Astrophys. J. 818:148. doi: 10.3847/0004-637X/818/2/148

Liu, R., Liu, C., Wang, S., Deng, N., and Wang, H. (2010). Sigmoid-to-flux-rope transition leading to a loop-like coronal mass ejection. Astrophys. J. Lett. 725, L84-L90. doi: 10.1088/2041-8205/725/1/L84

Liu, Z., Xu, J., Gu, B.-Z., Wang, S., You, J.-Q., Shen, L.-X., et al. (2014). New vacuum solar telescope and observations with high resolution. Res. Astron. Astrophys. 14, 705-718. doi: 10.1088/1674-4527/14/6/009

Myers, C. E., Yamada, M., Ji, H., Yoo, J., Jara-Almonte, J., and Fox, W. (2017). Quasi-static and dynamic magnetic tension forces in arched, line-tied magnetic flux ropes. Plasma Phys. Control. Fusion 59:014048. doi: 10.1088/0741-3335/59/1/014048

Priest, E. R. and Longcope, D. W. (2017). Flux-rope twist in eruptive flares and CMEs: due to zipper and main-phase reconnection. Sol. Phys. 292:25. doi: 10.1007/s11207-016-1049-0

Sasso, C., Lagg, A., and Solanki, S. K. (2014). Magnetic structure of an activated filament in a flaring active region. Astron. Astrophys. 561:A98. doi: 10.1051/0004-6361/201322481

Sharykin, I. N., Sadykov, V. M., Kosovichev, A. G., Vargas-Dominguez, S., and Zimovets, I. V. (2017). Flare energy release in the lower solar atmosphere near the magnetic field polarity inversion line. Astrophys. J. 840:84. doi: $10.3847 / 1538-4357 /$ aa6dfd

Tanaka, K. (1991). Studies on a very flare-active delta group - Peculiar delta SPOT evolution and inferred subsurface magnetic rope structure. Sol. Phys. 136, 133-149. doi: 10.1007/BF00151700

Toriumi, S. and Takasao, S. (2017). Numerical simulations of flare-productive active regions: $\delta$-sunspots, sheared polarity inversion lines, energy storage, and predictions. Astrophys. J. 850:39. doi: 10.3847/1538-4357/ aa95c2

Tritschler, A., Rimmele, T. R., Berukoff, S., Casini, R., Kuhn, J. R., Lin, H., et al. (2016). Daniel K. inouye solar telescope: high-resolution observing of the dynamic Sun. Astron. Nachrich. 337:1064. doi: 10.1002/asna.201612434

van Ballegooijen, A. A. and Martens, P. C. H. (1989). Formation and eruption of solar prominences. Astrophys. J. 343, 971-984.

Wang, H., Cao, W., Liu, C., Xu, Y., Liu, R., Zeng, Z., et al. (2015). Witnessing magnetic twist with high-resolution observation from the 1.6-m New Solar Telescope. Nat. Commun. 6:7008. doi: 10.1038/ncomms8008

Wang, H., Jing, J., Tan, C., Wiegelmann, T., and Kubo, M. (2008). Study of magnetic channel structure in active region 10930. Astrophys. J. 687, 658-667. doi: $10.1086 / 592082$

Wang, H., Liu, C., Ahn, K., Xu, Y., Jing, J., Deng, N., et al. (2017). High-resolution observations of flare precursors in the low solar atmosphere. Nat. Astron. 1:0085. doi: 10.1038/s41550-017-0085

Wang, H., Liu, R., Li, Q., Liu, C., Deng, N., Xu, Y., et al. (2018a). Extending counterstreaming motion from an active region filament to a sunspot light bridge. Astrophys. J. Lett. 852:L18. doi: 10.3847/2041-8213/aaa2f4

Wang, H., Yurchyshyn, V., Liu, C., Ahn, K., Toriumi, S., and Cao, W. (2018b). Strong transverse photosphere magnetic fields and twist in light bridge dividing delta sunspot of active region 12673. Res. Notes Am. Astron. Soc. 2:8. doi: 10.3847/2515-5172/aaa670

Xue, Z., Yan, X., Yang, L., Wang, J., and Zhao, L. (2017). Observing formation of flux rope by tether-cutting reconnection in the sun. Astrophys. J. Lett. 840:L23. doi: 10.3847/2041-8213/aa7066

Yan, X. L., Jiang, C. W., Xue, Z. K., Wang, J. C., Priest, E. R., Yang, L. H., et al. (2017). The eruption of a small-scale emerging flux rope as the driver of an M-class flare and of a coronal mass ejection. Astrophys. J. 845:18. doi: 10.3847/1538-4357/aa7e29

Yan, X. L., Xue, Z. K., Pan, G. M., Wang, J. C., Xiang, Y. Y., Kong, D. F., et al. (2015). The formation and magnetic structures of active-region filaments observed by NVST, SDO, and hinode. Astrophys. J. Suppl. Ser. 219:17. doi: 10.1088/0067-0049/219/2/17

Yang, S., Zhang, J., Liu, Z., and Xiang, Y. (2014). New vacuum solar telescope observations of a flux rope tracked by a filament activation. Astrophys. J. Lett. 784:L36. doi: 10.1088/2041-8205/784/2/L36

Zhang, J., Cheng, X., and Ding, M.-D. (2012). Observation of an evolving magnetic flux rope before and during a solar eruption. Nat. Commun. 3:747. doi: $10.1038 /$ ncomms 1753

Zirin, H., and Wang, H. (1993). Narrow lanes of transverse magnetic field in sunspots. Nature 363, 426-428. doi: 10.1038/363426a0

Conflict of Interest Statement: The authors declare that the research was conducted in the absence of any commercial or financial relationships that could be construed as a potential conflict of interest.

Copyright (C) 2019 Wang and Liu. This is an open-access article distributed under the terms of the Creative Commons Attribution License (CC BY). The use, distribution or reproduction in other forums is permitted, provided the original author(s) and the copyright owner(s) are credited and that the original publication in this journal is cited, in accordance with accepted academic practice. No use, distribution or reproduction is permitted which does not comply with these terms. 